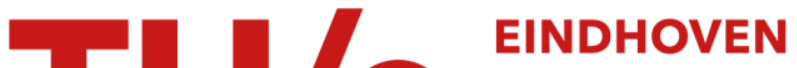 UNIVERSITY OF TECHNOLOGY
}

\section{In-situ measurement of dust charge density in nanodusty plasma}

Citation for published version (APA):

Staps, T. J. A., Donders, T. J. M., Platier, B., \& Beckers, J. (2022). In-situ measurement of dust charge density in nanodusty plasma. Journal of Physics D: Applied Physics, 55(8), [08LT01]. https://doi.org/10.1088/13616463/ac3581

DOI:

10.1088/1361-6463/ac3581

Document status and date:

Published: 24/02/2022

Document Version:

Publisher's PDF, also known as Version of Record (includes final page, issue and volume numbers)

Please check the document version of this publication:

- A submitted manuscript is the version of the article upon submission and before peer-review. There can be important differences between the submitted version and the official published version of record. People interested in the research are advised to contact the author for the final version of the publication, or visit the $\mathrm{DOI}$ to the publisher's website.

- The final author version and the galley proof are versions of the publication after peer review.

- The final published version features the final layout of the paper including the volume, issue and page numbers.

Link to publication

\section{General rights}

Copyright and moral rights for the publications made accessible in the public portal are retained by the authors and/or other copyright owners and it is a condition of accessing publications that users recognise and abide by the legal requirements associated with these rights.

- Users may download and print one copy of any publication from the public portal for the purpose of private study or research.

- You may not further distribute the material or use it for any profit-making activity or commercial gain

- You may freely distribute the URL identifying the publication in the public portal.

If the publication is distributed under the terms of Article $25 \mathrm{fa}$ of the Dutch Copyright Act, indicated by the "Taverne" license above, please follow below link for the End User Agreement:

www.tue.nl/taverne

Take down policy

If you believe that this document breaches copyright please contact us at:

openaccess@tue.nl

providing details and we will investigate your claim. 
LETTER • OPEN ACCESS

\section{In-situ measurement of dust charge density in nanodusty plasma}

To cite this article: Tim Jacobus Adrianus Staps et al 2022 J. Phys. D: Appl. Phys. 5508 LT01

View the article online for updates and enhancements.
You may also like

- Parametrization of current-voltage
$\frac{\text { characteristics and operation domains of }}{\text { cylindrical emissive probes in collisionless }}$
$\frac{\text { Maxwellian plasmas at rest }}{\text { S Shahsavani, X Chen and G Sanchez- }}$
Arriaga
- CHARGING AND COAGULATION OF
DUST IN PROTOPLANETARY PLASMA
ENVIRONMENTS
L. S. Matthews, V. Land and T. W. Hyde
- Dust charge measurement in a strongly
coupled dusty plasma produced by an rf
$\frac{\text { discharge }}{\text { S K Sharma, Ranjan Kalita, Y Nakamura }}$
et al.

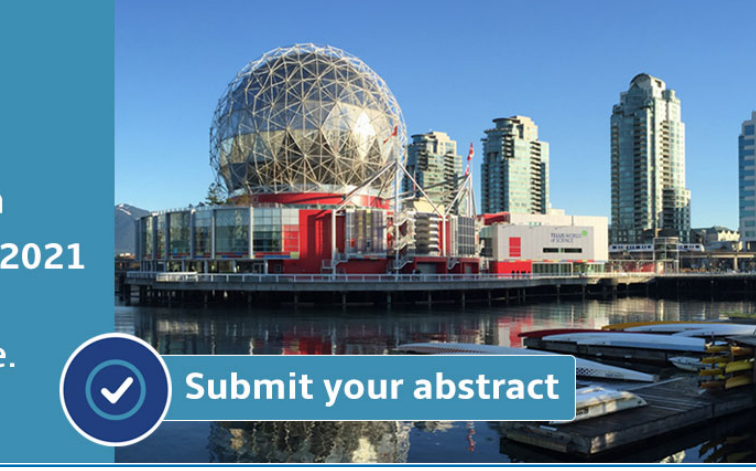




\title{
Letter
}

\section{In-situ measurement of dust charge density in nanodusty plasma}

\author{
Tim Jacobus Adrianus Staps* (D), Tim Jacobus Maria Donders $(\mathbb{D}$, \\ Bart Platier (i) and Job Beckers \\ Department of Applied Physics, Eindhoven University of Technology, PO Box 513, 5600MB Eindhoven, \\ The Netherlands \\ E-mail: t.j.a.staps@tue.nl
}

Received 14 September 2021, revised 25 October 2021

Accepted for publication 2 November 2021

Published 12 November 2021

\begin{abstract}
A dust grain immersed in a low-pressure gas discharge obtains a permanent negative surface charge due to the high mobility of electrons compared to that of ions. This charge essentially governs all fundamental processes in dusty and complex plasmas involving dust grains, neutrals, (an)ions and electrons and-consequently-virtually all industrial applications of these types of plasmas are affected and steered by it. In this work, we have measured the surface charge by application of laser-induced electron detachment from nanosized dust grains in concert with microwave cavity resonance spectroscopy and laser light extinction. The main result is that the electron release is governed by photodetachment rather than by thermionic emission, and that recharging of the dust grains occurs on timescales that are well in agreement with the orbital-motion-limited (OML) theory. The total surface charge density residing on the dust grains inside the laser volume follows from the saturation of the photodetachment signal, which was used in combination with dust density values derived from extinction measurements to estimate the mean dust charge. The negative dust charge on the $140 \mathrm{~nm}$ (average) diameter dust grains in this work is obtained to be in the range of $273-2519$ elementary charges, of which the lower bound matches well with analytical predictions using the OML theory.
\end{abstract}

Supplementary material for this article is available online

Keywords: nanodusty, dust charge, particle charge, nanoparticle, low-pressure dusty plasma, plasma charging, OML theory

(Some figures may appear in colour only in the online journal)

\section{Introduction}

Complex and dusty plasmas comprise a mixture of (partially) ionized gas with nano- to micrometer sized dust grains $[1,2]$,

* Author to whom any correspondence should be addressed.

Original Content from this work may be used under the terms of the Creative Commons Attribution 4.0 licence. Any further distribution of this work must maintain attribution to the author(s) and the title of the work, journal citation and DOI. and occur frequently in a wide variety of mature industrial processes and research niches such as semiconductor manufacturing [3, 4], nuclear fusion [5], and (functional) materials embedding nanoparticles [6-8]. The non-equilibrium between electron and heavy species temperatures provides a key advantage for low-temperature plasmas over conventional alternatives, resulting in (permanently and) negatively charged surfaces of, e.g. the reactor walls, electrodes and dust grains. For complex and dusty plasmas, the electric charge retained on the dust grains reflects a key parameter dictating fundamental processes such as Coulomb interactions-e.g. crystallization, 
instabilities, and Coulomb-induced collisions-in plasmalevitated dust crystals and dusty plasmas [9-14], dust grain formation by nucleation, coagulation, and accretion [15-20], void formation and laser-induced coalescence [21-25]. For applications, the effects of dust can be detrimental to, e.g. fusion energy reactors $[3,26]$ and plasmas created in semiconductor manufacturing $[4,27]$, which led to recent work focused on contamination control using plasmas [28-30]. The charge state of the dust particles in contact with the plasma is often a decisive factor in the dust retention mechanism. Therefore, it is of utmost importance, for current and future technologies and for plasma science, to understand the elementary charging processes driving dust-plasma interaction.

The floating potential — or the surface charge — acquired by plasma-embedded dust grains arises from the balance between the electron and ion currents, which results in permanently negatively charged dust grains for dust radii $a \gtrsim 10 \mathrm{~nm}$ under typical dusty plasma conditions $[31,32]$. The orbital-motionlimited (OML) theory is the governing theoretical framework predicting the floating potential of dust grains [33,34], which has been extended with numerous advancements to include, e.g. the effect of ion-neutral collisions [35-37]. The theory has been successfully applied to microparticle charging in different plasma regions such as the plasma bulk $[38,39]$, the plasma sheath [40], and the spatial afterglow [41]. On the contrary, only a limited number of works provide experimental data on the charge of nanosized dust grains embedded in plasmas [24, 42-44]. This lack of experimental data is mainly caused by the fact that most diagnostics for obtaining information about the dust grain charge are based on optical principles which dramatically suffer from a decreasing signal when the grain size becomes smaller than the wavelength of the light used for the diagnostic [45-47].

In this letter, laser-induced electron detachment from nanometer-sized dust grains is demonstrated experimentally in a low-pressure dusty plasma using ultraviolet (UV) laser pulses in order to close the gap in experimental data. The dust grains were formed in-situ by a typical gas discharge in an argon-hexamethyldisiloxane (HMDSO) gas mixture, and thereafter stably confined to the same volume in an argononly discharge. The change in free electron density caused by the laser-dust interaction was consecutively measured with ns-time resolution using time-resolved microwave cavity resonance spectroscopy (MCRS) [24, 48, 49]. The experimental dust charge was estimated by the ratio of the maximum free electron density released by the laser to the dust density obtained from laser light extinction measurements (see section A in Supplementary Information (available online at stacks.iop.org/JPD/55/08LT01/mmedia)).

This letter is organized as follows. First, the experimental procedure for dust growth and laser-induced electron detachment are explained in detail. Second, the time-resolved electron density providing three important observations is discussed and analyzed in detail with respect to the current physical understanding of photodetachment. Third, the measured dust charge density is used to estimate the dust charge, and the result is discussed in view of the current understanding in the literature. Additional information regarding elements of the results, discussion and interpretation can be found in the Supplementary Information file.

\section{Method}

In figure 1, a schematic top-view of the experimental setup is shown. Inside a vacuum vessel, a low-pressure plasma was created in a stainless steel hollow pillbox cavity, driven by a radiofrequency signal $\left(f_{\mathrm{RF}}=13.56 \mathrm{MHz}\right)$ with a plasma power of $P_{\mathrm{RF}}=20 \mathrm{~W}$. During the experiments described in this Letter, argon (with a partial pressure of $p_{\mathrm{Ar}}=5.4 \times 10^{-2} \mathrm{mbar}$ ) was admixed with HMDSO ( $p_{\text {HMDSO }}=1.6 \times 10^{-2}$ mbar), of which the latter was the precursor to dust grain formation. These dust grains gained a permanent negative charge after reaching a size larger than $\sim 10 \mathrm{~nm}[31,32]$, enabling them to remain trapped in the discharge volume. A detailed description of the microwave cavity with integrated discharge electrodes and the dust collection system can be found in section B in Supplementary I.

The complete experiment consisted of three separate stages. During the growth phase, an Ar/HMDSO plasma was generated for $60 \mathrm{~s}$. Afterwards, the HMDSO flow was terminated, and the nanodusty plasma entered the settling stage, in which the plasma adjusted to the change in pressure, until the dust cloud found a steady state in terms of size and density. It should be noted that the electron density was strongly depleted during the coagulation phase, as a consequence of the permanent negative charging of the dust cloud, and remained depleted during subsequent experiments. Finally, the measurement stage consisted of six separate experiments with varying laser pulse energy. In each experiment, UV laser pulses were fired with a frequency of $f_{\text {laser }}=20 \mathrm{~Hz}$, and the microwave response was temporally recorded per pulse. This microwave response, i.e. the change in resonance frequency of the used resonant mode, was caused by the additional photodetached electrons upon laser irradiation. The global electron density of the (dusty) plasma and laser light transmission through the dust cloud were monitored over the course of all three stages of the experiment, of which the signals can be found in section $\mathrm{C}$ in Supplementary Information.

For the actual laser-induced electron detachment experiments, a frequency-quadrupled (UV) laser (Quantel BigSky Ultra, wavelength $\lambda_{\text {ph }}=266 \mathrm{~nm}$ ) was used to generate short ( $\sim 8 \mathrm{~ns}$ ) pulses which traveled through the cavity via a thin slit on each side. Upon interaction with the laser photons, electrons were released from the dust grains, and therefore, the free electron density of the plasma was temporally increased. It was verified, in vacuum at a pressure of $2 \times 10^{-7} \mathrm{mbar}$ and with a pristine argon discharge, that no detectable amount of electrons was released from the metal surfaces of the cavity and that photo-ionization did not result in a cavity response exceeding the noise band. The volume-averaged density of these free electrons was measured by means of MCRS, as previously applied to a multitude of plasma types $[6,24,48-51]$. To this end, a microwave generator (Stanford Research Systems Model SG386) sent electromagnetic waves with a fixed frequency into the cavity. This microwave 


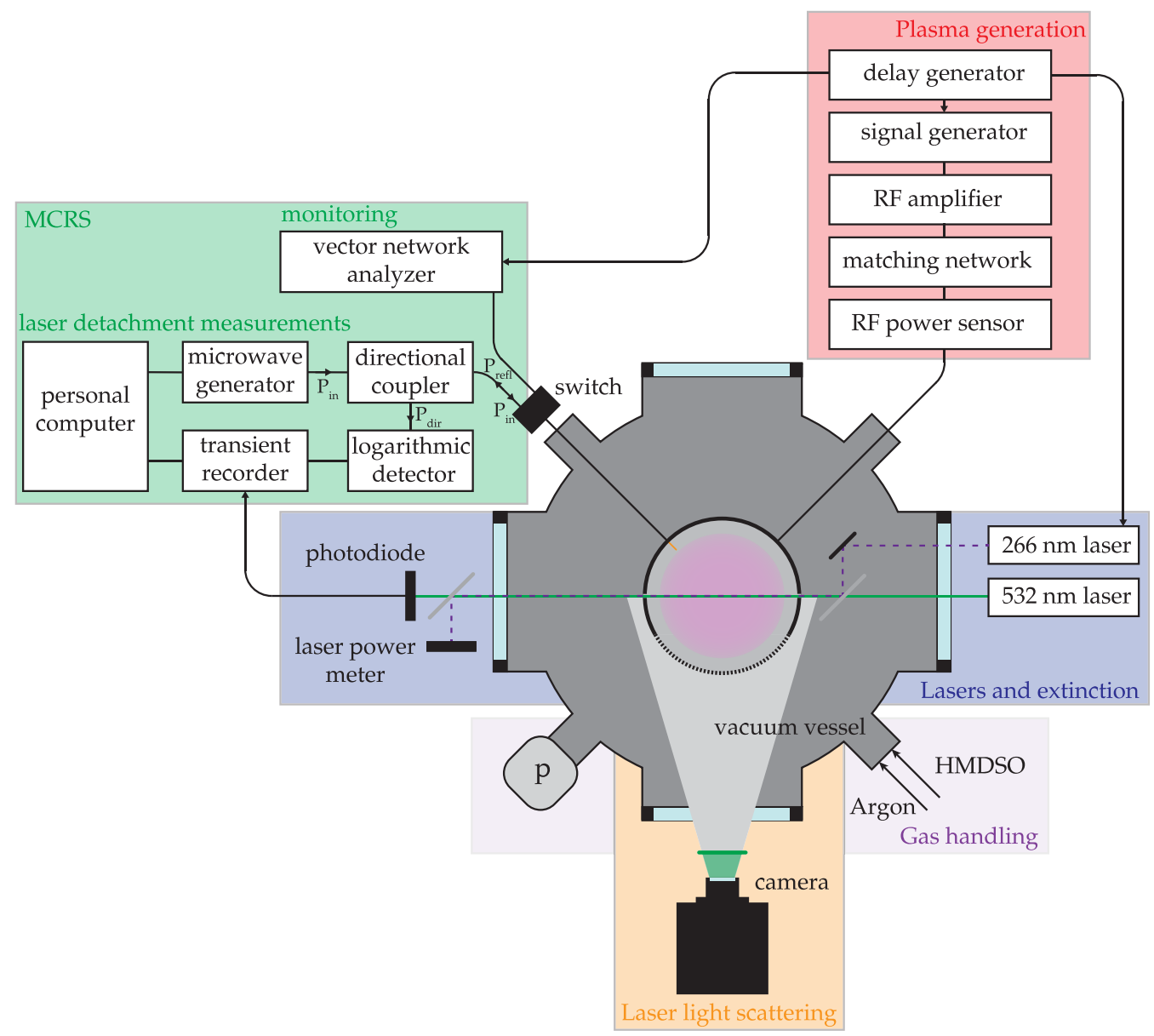

Figure 1. Schematic overview of the experimental setup used for creating, monitoring and probing the nanodusty plasma. The symbol $\mathrm{P}$ indicates pressure sensor.

radiation had a fixed power of only $P_{\mu \mathrm{w}}=40 \mathrm{~mW} \ll P_{\mathrm{RF}}$ meaning that the microwaves were non-intrusive. A part of the microwave power was absorbed inside the cavity while the rest was reflected. Ten percent of the reflected power was sent to a logarithmic detector (Analog Devices HMC602LP4) using a directional coupler (Mini-Circuits ZHDC-16-63+). The detector voltage was eventually sampled using a transient recorder (Spectrum M3i.4121-exp) with a sample frequency of $250 \mathrm{MHz}$ for a range of microwave frequencies close to the resonance frequency. In this way, the spectrum around a resonant eigenmode of the microwave cavity could be retrieved in a temporally-resolved fashion.

The frequency at which microwaves in the cavity are at resonance depends on the permittivity of the medium inside the cavity. This introduces a relation between the shift in resonance frequency $\Delta f$ and the (change in) electron density $n_{\mathrm{e}}$, as derived from the Slater perturbation theorem [52-54],

$$
n_{\mathrm{e}}(t)=\frac{8 \pi^{2} \varepsilon_{0} m_{\mathrm{e}} f_{\mathrm{p}}^{2}(t)}{e^{2} \mathcal{V}} \frac{\Delta f(t)}{f_{1}},
$$

with $n_{\mathrm{e}}, m_{\mathrm{e}}$ and $e$ the electron density, mass and (elementary) charge, $\varepsilon_{0}$ the vacuum permittivity, $f_{\mathrm{p}}$ the resonance frequency with momentary perturbation, $f_{1}$ the resonance frequency without the perturbation, and $\Delta f=f_{\mathrm{p}}-f_{1}$ the shift in resonance frequency. The parameter $\mathcal{V}$ is defined as the (microwave-)electric-field-weighted ratio of the laser beam $V_{\mathrm{b}}$ to cavity volume $V_{\mathrm{c}}$ as

$$
\mathcal{V}=\frac{\iiint_{V_{\mathrm{b}}}|\mathbf{E}(\mathbf{r})|^{2} d^{3} \mathbf{r}}{\iiint_{V_{\mathrm{c}}}|\mathbf{E}(\mathbf{r})|^{2} d^{3} \mathbf{r}} .
$$

Here, the microwave electric field $\mathbf{E}(\mathbf{r})$ for the $\mathrm{TM}_{010}$ resonant mode was computed using a numerical model of the microwave cavity in COMSOL Multiphysics ${ }^{\circledR}$ (see section D in Supplementary Information). Subsequently, the electricfield-weighted volume ratio $\mathcal{V}$ was calculated by evaluating equation (2) numerically using the measured laser beam diameter $D_{\mathrm{b}}$, the cavity diameter $D_{\mathrm{c}}=66 \mathrm{~mm}$ and height $H_{\mathrm{c}}=$ $40 \mathrm{~mm}$, and the numerical solution for the microwave electric field E. Details on the microwave electric field, the volume ratio and the position of the laser beam in the cavity can be found in section D in Supplementary Information.

In order to obtain a sufficient signal-to-noise ratio, a typical measurement consisted of a number of time series $N_{\text {meas }}=$ $N_{\text {freq }} N_{\text {av }}$, with $N_{\text {freq }}$ the number of probed frequencies and $N_{\text {av }}$ the number of averages per frequency. It was therefore important that the plasma and dust conditions remained as stable as possible during the complete measurement set. Several diagnostics were implemented in order to globally monitor both 
the dust growth process and the stability of the sample thereafter (see measurement results in section $C$ in Supplementary Information). First, global electrical parameters of the RF discharge, such as the plasma power and the phase angle between RF voltage and current, were tracked by an Impedans OctivPoly 1.0 power sensor. Additionally, the dust cloud was visualized by laser light scattering using a green $(532 \mathrm{~nm})$ laser sheet. The scattered light was detected by a Photron Mini UX100 FastCam with a laser line filter, while the transmitted light was monitored by a photodiode to perform extinction measurements. Furthermore, a vector network analyzer (VNA) was used to perform spectral scans of the microwave response at low temporal resolution $(\sim 100 \mathrm{~ms})$, so that the electron density could still be monitored, even when the transient recorder-based MCRS system was not activated. The timing of the experiment was controlled by a BNC 577 Series pulse/delay generator, which sent trigger pulses to the VNA and the pulsed UV laser. The output of the pulsed UV laser was used as a trigger for the transient recorder such that jitter in the laser output was inherently corrected for.

\section{Results}

Figure 2 depicts the measured laser-induced change of the electron density as a function of time, which has been measured for different laser pulse energies as indicated in the figure's legend. For each laser pulse energy, the time-resolved signals clearly show three distinct phases in the evolution of the free electron density. The reference value for the resonance frequency $f_{1}$ was obtained from phase (I), during which the dusty plasma was undisturbed. At the beginning of phase (II), i.e. $t=0 \mu \mathrm{s}$, the laser was shot and, subsequently, the free electron density increased rapidly within about $70 \mathrm{~ns}$. After reaching a peak value at the end of phase (II), the laser-induced electron density decreased gradually following an exponential decay back to the steady state value observed in phase (I).

From the results presented in figure 2, three distinct observations can be made:

- The fast rise time $\tau_{\text {rise }}$ during phase (II) is comparable to the fundamental cavity response time expected for an instantaneous change in the free electron density [55]. $\tau_{\text {rise }} \approx 3 \tau_{\text {cavity }}$ and $\tau_{\text {cavity }}=Q_{1} /\left(2 \pi f_{1}\right) \approx 18$ ns. This limited response time of a microwave resonant cavity is due to a minimum time the cavity-system needs in order to load or decharge the microwave energy associated with the excited resonant mode.

- As can be seen from the inset in figure 2, the peak value of the laser-induced electron density increases for increasing laser pulse energy, and saturates for sufficiently high laser pulse energies.

- The density of additionally released electrons decays exponentially in phase (III), of which the time constant is estimated to be $\tau_{\text {decay }} \approx 1.6-2.1 \mu$ s based on an exponential fit through the experimental data.

Besides the above mentioned observations, it is absolutely clear that a significant amount of free electrons is released upon irradiation of the dust cloud with the UV laser. In general, there are four widely accepted physical mechanisms that could explain the decharging of plasma-embedded dust grains.

Field emission [2], which is the release of electrons due to a strong electric field, is only important for dust grain radii larger than a micrometer whereas in our experiments the grain size is roughly a few hundred nanometers (see section $\mathrm{E}$ in Supplementary Information).

Secondary electron emission $[34,56]$, which is the release of electrons from the material after impact by high-energy electrons, is only relevant for high-energy electrons of which the density is very low under the conditions considered.

The two other mechanisms-i.e. thermionic emission $[57,58]$ and photon-induced electron detachment (also called photodetachment) [59-62]—may be considered more likely to become dominant under the current conditions.

Here, thermionic emission is caused by a high surface temperature (possibly caused by laser irradiation) transferring thermal energy to electrons such that they can overcome the work function, while in the case of photodetachment this energy is provided in the form of photon energy directly. It is due to this difference that the effect of photodetachment events - in contrast to the situation for thermionic emissionmay be considered instantaneous compared to the sampling time [63]. To investigate which of the two latter mechanisms is dominantly responsible for the observed release of electrons, a model for thermionic emission, i.e. a coupled model for predicting the changes in dust grain temperature and the dust grain floating potential upon laser irradiation, has been constructed from which typical (de-)charging timescales can be retrieved (see section $\mathrm{F}$ and $\mathrm{G}$ in Supplementary Information). Under the current experimental conditions, it can be concluded from this model that the floating potential hardly changes due to the vast increase in the dust grain's temperature. This indicates thatunder the current conditions-laser-induced photodetachment dominates over laser-induced thermionic emission. Moreover, this dominant contribution of photodetachment to the overall electron release process is supported by all of the three observations mentioned earlier as discussed further on.

The first observation was that the signal grows during phase (II) up to the peak value within approximately $3 \tau_{\text {cavity }}$. As photodetachment occurs almost instantaneously for the duration of the laser shot ( $\sim 8 \mathrm{~ns})$ [63], the signal reflects the cavity response to a sudden perturbation as described by Van Ninhuijs et al [55]:

$$
\Delta f_{\mathrm{m}}=\Delta f\left(1-\exp \left(-\frac{t}{\tau_{\text {cavity }}}\right)\right),
$$

with $\Delta f_{\mathrm{m}}$ the measured frequency shift, and $\Delta f$ the actual frequency shift. By contrast, thermionic emission exhibits a rise time of about $0.6 \mu \mathrm{s}$ in an extreme case with low work function of the dust grain. As a consequence, the rise time strongly indicates that photodetachment is responsible for the release of electrons.

The second observation strongly suggested that the total released electron density saturated for increasing laser pulse energy (see the inset in figure 2). From literature, it is known 


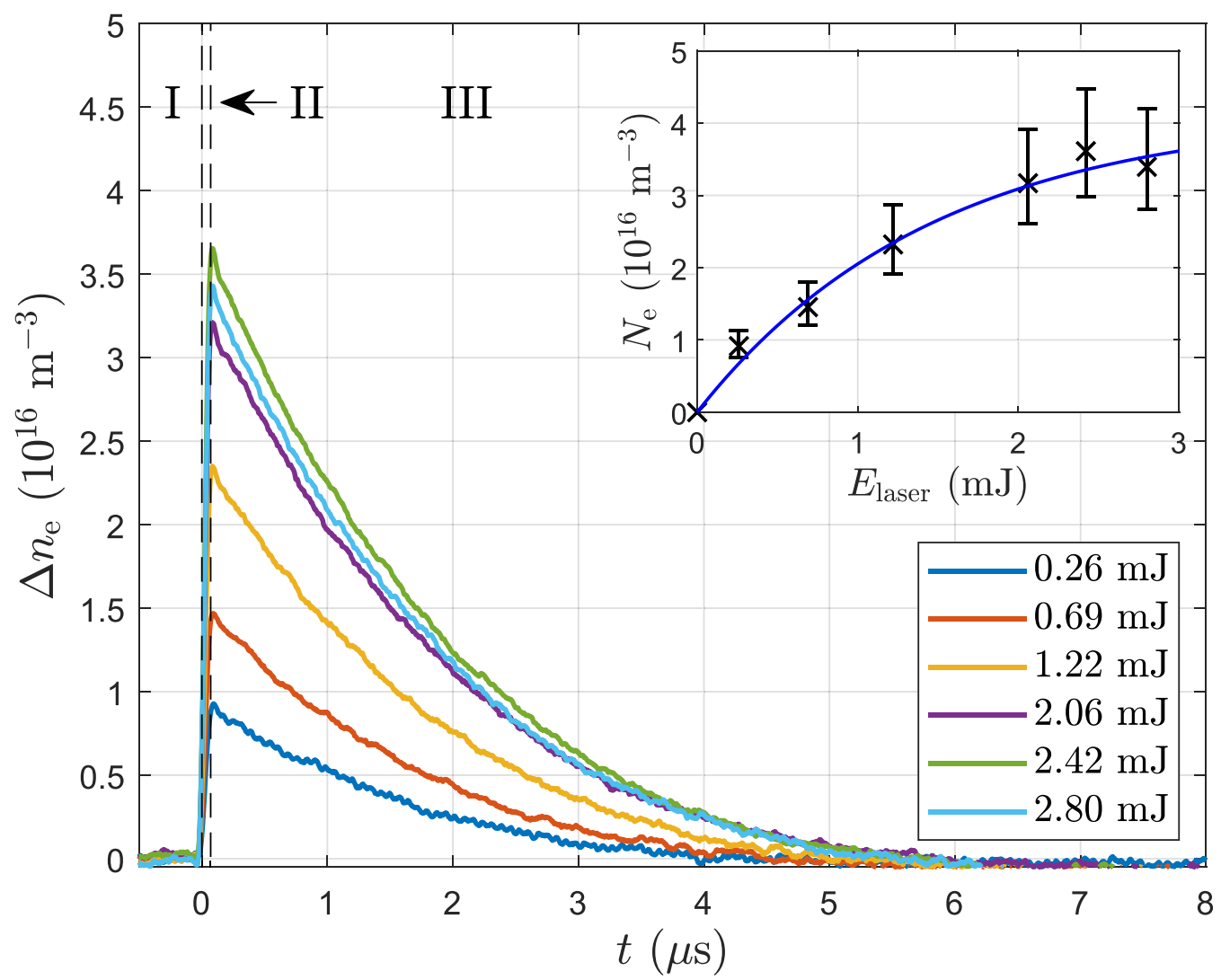

Figure 2. Time-resolved laser-induced electron density for different values of laser pulse energy, as defined by the colors/legend. The inset depicts the peak value of the laser-released electron density for each laser experiment.

that the number of electrons $N_{\mathrm{e}}$ released by photodetachment saturates for sufficiently high laser pulse energies following an exponential curve $[60,64,65]$ :

$$
N_{\mathrm{e}}=\hat{N}_{\mathrm{e}}\left(1-\exp \left(-\frac{\sigma_{\mathrm{pd}} E_{\mathrm{laser}}}{E_{\mathrm{ph}} S}\right)\right)
$$

Here, the laser pulse energy is represented by $E_{\text {laser }}$, the photon energy is denoted by $E_{\mathrm{ph}}=4.66 \mathrm{eV}$ (i.e. $\lambda_{\mathrm{ph}}=266 \mathrm{~nm}$ ), and the laser cross section $S=0.031 \mathrm{~cm}^{2}$. Fitting equation (4) to the saturation data yields an asymptotic value of $\hat{N}_{\mathrm{e}}=$ $4.15 \times 10^{16} \mathrm{~m}^{-3}$ and photodetachment cross section $\sigma_{\mathrm{pd}}=$ $1.6 \times 10^{-17} \mathrm{~m}^{2}$. Consequently, there are two interesting notes with respect to the saturation study. First, the photodetachment cross section is much smaller than the hard sphere cross section, based on the dust size, $\sigma \sim \pi a^{2} \approx 6 \times 10^{-14}$ $\mathrm{m}^{2}$ by three orders of magnitude. Second, note that if laserinduced thermionic emission would have been dominant, an opposite curvature of this curve would have been expected. This is because, with thermionic emission being the dominant electron release mechanism, the dependence of the density of released electrons on the laser pulse energy would have been stronger than linear (see section G in Supplementary Information).

The third observation concerned the temporal decay of the released electron densities back to their quasi-steady state values. Based on the OML theory, the timescale on which the electron and ion currents restore the equilibrium floating potential of the dust grains can be calculated from the following equation, which has been derived in section $\mathrm{H}$ in Supplementary Information from the electron and ion current:

$$
\tau_{\text {charging }}=\left(\frac{e^{2} a n_{\mathrm{i}} v_{\mathrm{T}_{\mathrm{i}}}}{4 \varepsilon_{0} k_{\mathrm{B}} T_{\mathrm{e}}}\left(\frac{T_{\mathrm{e}}}{T_{\mathrm{i}}}+\frac{n_{\mathrm{e}} v_{\mathrm{T}_{\mathrm{e}}}}{n_{\mathrm{i}} v_{\mathrm{T}_{\mathrm{i}}}} \exp \left(\frac{e V_{\mathrm{d}}}{k_{\mathrm{B}} T_{\mathrm{e}}}\right)\right)\right)^{-1} .
$$

Here, $T_{\mathrm{e}}$ denotes the electron temperature, $a$ the dust grain radius, $n_{\mathrm{i}}$ the ion density, and $T_{\mathrm{i}}$ the ion temperature. It should be noted that this equation has been derived considering that $n_{\mathrm{e}} \neq n_{\mathrm{i}}$, whereas in other works the charging timescale is often given for fixed ratio of $T_{\mathrm{e}} / T_{\mathrm{i}}$ or for $n_{\mathrm{e}}=n_{\mathrm{i}}$ [2, 34, 66-68]. The plasma and dust parameters used for the evaluation of the charging timescale are as follows: $T_{\mathrm{e}}=1-3 \mathrm{eV}, n_{\mathrm{e}}=$ $2 \times 10^{15} \mathrm{~m}^{-3}$ (equal to $n_{\mathrm{e}}$ for $t \geq 70 \mathrm{~s}$, see section C in Supplementary Information), $T_{\mathrm{i}}=0.026 \mathrm{eV}, n_{\mathrm{i}}=9.2 \times 10^{15} \mathrm{~m}^{-3}-$ $4.15 \times 10^{16} \mathrm{~m}^{-3}$, and $a=140 \mathrm{~nm}$ (obtained by SEM images, see section $\mathrm{E}$ in Supplementary Information). The range for the ion density is motivated by using a lower limit equal to the electron density during the nucleation phase without electron depletion $\left(\check{n}_{\mathrm{i}}=9.2 \times 10^{15} \mathrm{~m}^{-3}\right.$, equal to $n_{\mathrm{e}}$ for $t \leq 30 \mathrm{~s}$, see section $C$ in Supplementary Information), and assuming that the ion density does not change during the subsequent phases. However, it is likely that the ion density increases due to an increase in the electron mean energy, which balances the decrease in ionization caused by electron depletion due to the permanent charging of dust particles. Therefore, 
we assume an upper limit equal to the total dust charge density measured plus the free electron density of the discharge $\left(\hat{n}_{\mathrm{i}}=4.15 \times 10^{16} \mathrm{~m}^{-3}\right.$, i.e. equal to $\left.\hat{N}_{\mathrm{e}}+n_{\mathrm{e}}\right)$. This upper limit is motivated by the fact that quasi-neutrality should be obeyed, and hence, that the ion density is balanced by the total dust charge density. Due to electron depletion, the free electron density is much lower than the contribution of the negatively charged dust particles to the quasi-neutrality condition. Under these conditions, the charging timescale ranges between $1.3 \mu \mathrm{s}$ $\left(T_{\mathrm{e}}=1 \mathrm{eV}\right.$ and $\left.n_{\mathrm{i}}=4 \times 10^{16} \mathrm{~m}^{-3}\right)$ and $4.4 \mu \mathrm{s}\left(T_{\mathrm{e}}=3 \mathrm{eV}\right.$ and $\left.n_{\mathrm{i}}=9.2 \times 10^{15} \mathrm{~m}^{-3}\right)$. It should be noted that a change in the ion density dominates over the effect of a change in electron temperature, and thus it is important to account for the fact that $n_{\mathrm{e}} \neq n_{\mathrm{i}}$ in the evaluation of the charging timescale. Considering the measured $\tau_{\text {decay }}$, the experimental values agree well with the estimates for $\tau_{\text {charging. The charging timescale }}$ for heated dust grains is almost two orders of magnitude larger $(\tau \sim 100 \mu \mathrm{s})$ due to the thermionic emission current. As a consequence, the agreement with the timescale based on only the OML currents implies that the thermionic emission current is irrelevant. In conclusion, this observation suggests also that photodetachment is the dominant detachment process.

The total density of detached electrons can be used to estimate the dust charge, which equals $q_{\mathrm{d}}=\hat{N}_{\mathrm{e}} / n_{\mathrm{d}}$ in case of saturation-meaning that all electrons have been released from the dust grain. The assumption here is that the dust grains irradiated by the laser beam are mono-disperse, which seems a reasonable assumption based on the SEM images (see section E in Supplementary Information). The saturated electron density $\hat{N}_{\mathrm{e}}$ results from the measurement of the peak value of the electron density as a function of laser pulse energy, which involves three measurements errors estimated as $\Delta_{\Delta f}=\Delta_{f_{\mathrm{p}}}=3 \times 10^{5} \mathrm{~Hz}$ based on the noise band of $f_{\mathrm{p}}$, and $\Delta \mathcal{V}=1.4 \times 10^{-4}$ based on the uncertainty in the laser beam cross section (see section D in Supplementary Information). This implies that $\hat{N}_{\mathrm{e}}=3.0 \times 10^{16} \mathrm{~m}^{-3}-5.3 \times 10^{16} \mathrm{~m}^{-3}$ by linearization of equation (1) to calculate the error in $N_{\mathrm{e}}$ for $E_{\text {laser }}=2.80 \mathrm{~mJ}$. The dust density $n_{\mathrm{d}}$ is estimated from extinction measurements (see section A in Supplementary Information), which provides a dust density between $n_{\mathrm{d}}=2.1 \times 10^{13}$ $\mathrm{m}^{-3}$ and $n_{\mathrm{d}}=1.1 \times 10^{14} \mathrm{~m}^{-3}$, for dust radius $a=140 \mathrm{~nm}$, depending on the assumed material and its complex refractive index. As a consequence, the dust charge is estimated in the range $q_{\mathrm{d}} \approx 273-2519$ elementary charges.

\section{Discussion}

The dust charge density $q_{\mathrm{d}} n_{\mathrm{d}}=\hat{N}_{\mathrm{e}}=4.15 \times 10^{16} \mathrm{~m}^{-3}$ indicates that the number of dust-confined electrons largely exceeds the free electron density $\left(n_{\mathrm{e}}<2 \times 10^{15} \mathrm{~m}^{-3}\right.$ (see section C in Supplementary Information). This effect is captured by the Havnes parameter [46]:

$$
P=4 \pi \varepsilon_{0} a \frac{T_{\mathrm{e}}}{e} \frac{n_{\mathrm{d}}}{n_{\mathrm{i}}} \approx 0.2-3.2,
$$

given the same set of parameters as before, using $n_{\mathrm{d}}=2.1 \times$ $10^{13} \mathrm{~m}^{-3}-1.1 \times 10^{14} \mathrm{~m}^{-3}$ and $n_{\mathrm{i}}=1 \times 10^{16} \mathrm{~m}^{-3}-4 \times 10^{16}$ $\mathrm{m}^{-3}$. The dust charge measurements by Tadsen et al [42] were performed under conditions where the free electron density was severely depleted $(P \approx 10-50)$ and found much lower values for the dust charge, i.e. $q_{\mathrm{d}} \approx 10-60$ elementary charges. Although the dusty plasma considered in this work suffered from electron depletion, the effect was not as severe as in the work of, e.g. Tadsen et al [42] and Deka et al [44].

To account for electron depletion, the dust charge is evaluated theoretically using the OML theory with the density and temperature of the electrons and ions as before. Using the OML theory, the electron and ion current towards the dust grain with floating potential $V_{\mathrm{d}}$ are described as:

$$
\begin{gathered}
I_{\mathrm{e}}=-e \pi a^{2} n_{\mathrm{e}} v_{\mathrm{T}_{\mathrm{e}}} \exp \left(\frac{e V_{\mathrm{d}}}{k_{\mathrm{B}} T_{\mathrm{e}}}\right), \\
I_{\mathrm{i}}=e \pi a^{2} n_{\mathrm{i}} v_{\mathrm{T}_{\mathrm{i}}}\left(1-\frac{e V_{\mathrm{d}}}{k_{\mathrm{B}} T_{\mathrm{i}}}\right),
\end{gathered}
$$

where $v_{\mathrm{T}_{s}}=\sqrt{\frac{k_{\mathrm{B}} T_{s}}{2 \pi m_{s}}}$ with species $s=e, i$ for electrons and ions, respectively. Balancing equations (7) and (8), the floating potential of the dust grain provides a theoretical dust grain charge in the range:

$$
q_{\mathrm{d}}=4 \pi \varepsilon_{0} a V_{\mathrm{d}} \approx(-189 \mathrm{e})-(-386 \mathrm{e}),
$$

using the same (pure argon) plasma parameters as before $\left(T_{\mathrm{e}}=3 \mathrm{eV}, T_{\mathrm{i}}=0.026 \mathrm{eV}, n_{\mathrm{e}}=2 \times 10^{15} \mathrm{~m}^{-3}\right.$, $\left.n_{\mathrm{i}}=1 \times 10^{16} \mathrm{~m}^{-3}-4 \times 10^{16} \mathrm{~m}^{-3}, a=140 \mathrm{~nm}\right)$. For the purpose of comparison, the dust charge is also evaluated by setting $n_{\mathrm{e}}=4 \times 10^{16} \mathrm{~m}^{-3}$, which results in a theoretical charge of -688 e in case the electrons are not depleted. Consequently, the non-depleted OML charge $\left(q_{\mathrm{d}}=-688 \mathrm{e}\right)$ provides an upper bound for our measurement, and measurements performed under severe electron depletion provide a lower bound $\left(q_{\mathrm{d}}=(-10 \mathrm{e})-(-80 \mathrm{e})[42,44]\right)$. Moreover, this demonstrates that an accurate measurement of the dust density is necessary to eliminate the uncertainty in the dust charge in order to perform a quantitative comparison to the theory and literature.

There are two important adaptations to the basic OML theory that are applicable to the experimental conditions considered in this work. First, Tang and Delzanno [69] found that accounting for the ion density response to the presence of dust grains often leads to a significant increase of the dust charge, but this does not apply in our case because the dust grain radius is much smaller than the electron Debye length. Second, the effect of ion-neutral collisions is apparent for sufficiently small Knudsen numbers, where $\mathrm{Kn}_{R_{0}}=24$ under our conditions according to the definition by Gatti and Kortshagen [35]. This implies that the transition regime between the collisionless (OML) and the collision-enhanced regime is applicable, and that a charge reduction due to ion-neutral collisions can be expected. Nevertheless, the OML prediction seems to provide a reasonable expectation for the dust grain charge under the conditions considered in this work. 


\section{Conclusion}

In conclusion, the negative surface charge of dust grains immersed in a low-pressure dusty plasma was probed by exposing them to pulsed laser irradiation. As a consequence, laser-induced electron detachment caused a sudden change in the free electron density, which was measured time-resolved using MCRS. The results demonstrate that electrons were quickly removed from the dust grains due to photodetachment and, subsequently, that the dust grains recharged to their steady state charge on timescales predicted by OML theory. The total dust charge density was used in combination with the estimated number density of dust grains to calculate a mean dust charge. Comparing the experimental dust charge in the range $273-2519$ (elementary charges) to the OML theory, it was found that the OML prediction, i.e. $189-386$ (elementary charges), is close to the experimental lower bound. However, experimental findings by others $[42,44]$ are much lower due to severe electron depletion under their (different) conditions. More detailed knowledge of the complex refractive index (e.g. by ex-situ measurement of the refractive index of a collected dust sample) and radius such as measured by Groth et al [45] would lead to an improved accuracy of the measured dust charge, and consequently, facilitate a quantitative comparison and evaluation of the OML theory. For future experiments, we are currently investigating different options to measure the dust particle properties in-situ, such as the dust density and size, and to determine the refractive index ex-situ, which could be combined with dust charge density measurements for the accurate determination of the dust charge.

\section{Data availability statement}

The measurement data is available from the corresponding author upon reasonable request.

\section{Acknowledgments}

The authors wish to thank A.B. Schrader and P. Sanders for their skilful technical support during the preparation of the experiments. This activity is co-funded by PPS-contribution Research and Innovation of the Ministry of Economic Affairs and Climate Policy (The Netherlands), and Prodrive Technologies B.V.

\section{Competing interests}

The authors declare no competing interests.

\section{Code availability}

The computer code used for the data analysis and interpretation is available from the corresponding author upon reasonable request.

\section{ORCID iDs}

Tim Jacobus Adrianus Staps (1) https://orcid.org/0000-00029859-2942

Tim Jacobus Maria Donders (1) https://orcid.org/0000-00024671-8988

Bart Platier (10 https://orcid.org/0000-0003-4524-0131

Job Beckers (1) https://orcid.org/0000-0001-6116-7013

\section{References}

[1] Pustylnik M, Pikalev A, Zobnin A, Semenov I, Thomas H and Petrov O 2021 Contrib. Plasma Phys. e202100126 202100126

[2] Shukla P and Mamun A 2002 Introduction to Dusty Plasma Physics (Bristol: Institute of Physics Publishing Ltd)

[3] Merlino R L and Goree J A 2004 Phys. Today 57 32-38

[4] Beckers J, van de Ven T, van der Horst R, Astakhov D and Banine V 2019 Appl. Sci. 9 1-23

[5] Winter J 2000 Phys. Plasmas 7 3862-6

[6] Boufendi L, Jouanny M C, Kovacevic E, Berndt J and Mikikian M 2011 J. Phys. D: Appl. Phys. 44174035

[7] Hong J et al 2019 J. Mater. Chem. C 7 6369-74

[8] Jung W et al 2021 Nature 592 54-59

[9] Fortov V E and Petrov F 2010 High Temp. 48 943-56

[10] Maddox J 1994 Nature 370411

[11] Khrapak S and Morfill G 2009 Contrib. Plasma Phys. $49148-68$

[12] Gopalakrishnan R and Hogan C J 2012 Phys. Rev. E 85026410

[13] Filinov V S, Petrov O F, Fortov V E, Molotkov V I, Khakhaev A D and Podrjadchikov S F 2005 Contrib. Plasma Phys. 45 176-84

[14] Thomas H, Morfill G E, Demmel V, Goree J, Feuerbacher B and Möhlmann D 1994 Phys. Rev. Lett. 73 652-5

[15] Girshick S L 2020 J. Vac. Sci. Technol. A 38011001

[16] Belov I A, Ivanov A S, Ivanov D A, Pal A F, Starostin A N, Filippov A V, Dem'yanov A V and Petrushevich Y V 2000 J. Exp. Theor. Phys. 90 93-101

[17] Horanyi M and Goertz C K 1990 Astrophys. J. 361155

[18] Matthews L S, Land V and Hyde T W 2012 Astrophys. J. 7448

[19] Berndt J, Kovačević E, Stefanović I, Stepanović O, Hong S H, Boufendi L and Winter J 2009 Contrib. Plasma Phys. 49 107-33

[20] Marshall R S, Chai K B and Bellan P M 2017 Astrophys. J. 83756

[21] Mikikian M, Cavarroc M, Couëdel L, Tessier Y and Boufendi L 2010 Pure Appl. Chem. 82 1273-82

[22] Stefanovií I, Kovačević E, Berndt J and Winter J $2007 \mathrm{~J}$. Phys.: Conf. Ser. 71012015

[23] Stoffels W W, Stoffels E, Ceccone G and Rossi F 1999 J. Vac. Sci. Technol. A 17 3385-92

[24] Stoffels E, Stoffels W W, Kroesen G M W and de Hoog F J 1996 J. Vac. Sci. Technol. A 14 556-61

[25] Stoffels W W, Stoffels E, Kroesen G M W and de Hoog F J 1996 J. Vac. Sci. Technol. A 14 588-94

[26] Winter J, Fortov V E and Nefedov A P 2001 J. Nucl. Mater. 290-293 509-12

[27] Boufendi L and Bouchoule A 2002 Plasma Sources Sci. Technol. 11 A211

[28] van de Kerkhof M A, Galutschek E, Yakunin A, Cats S and Cloin C 2020 Proc. SPIE $11489114890 \mathrm{~K}$

[29] Beckers J, van Minderhout B, Blom P, Kroesen G and Peijnenburg T 2020 Proc. SPIE 11323 113232L

[30] van Huijstee J, van Minderhout B, Rompelberg R M H, Blom P, Peijnenburg T and Beckers J 2021 Proc. SPIE $11611116113 \mathrm{~A}$ 
[31] Bouchoule A and Boufendi L 1993 Plasma Sources Sci. Technol. 2 204-13

[32] Boufendi L, Bouchoule A, Porteous R K, Blondeau J P, Plain A and Laure C 1993 J. Appl. Phys. 73 2160-2

[33] Langmuir I 1928 Proc. Natl. Acad. Sci. USA 14 627-37

[34] Goree J 1994 Plasma Sources Sci. Technol. 3 400-6

[35] Gatti M and Kortshagen U 2008 Phys. Rev. E 78046402

[36] D'yachkov L G, Khrapak A G, Khrapak S A and Morfill G E 2007 Phys. Plasmas 14042102

[37] Salnikov M V, Fedoseev A V and Sukhinin G I 2019 J. Phys.: Conf. Ser. 1393012022

[38] Chaudhuri M, Khrapak S A and Morfill G E 2011 AIP Conf. Proc. 1397 263-4

[39] Ratynskaia S et al 2004 Phys. Rev. Lett. 93 8-11

[40] Beckers J, Ockenga T, Wolter M, Stoffels W W, Van Dijk J, Kersten H and Kroesen G M W 2011 Phys. Rev. Lett. 106115002

[41] Van Minderhout B, Van Huijstee J C A, Platier B, Peijnenburg T, Blom P, Kroesen G M W and Beckers J 2020 Plasma Sources Sci. Technol. 29065005

[42] Tadsen B, Greiner F, Groth S and Piel A 2015 Phys. Plasmas 22113701

[43] Smith M A, Goodrich J, Rahman H U and Mohideen U 2001 IEEE Trans. Plasma Sci. 29 216-20

[44] Deka T, Boruah A, Sharma S K and Bailung H 2017 Phys. Plasmas 24093706

[45] Groth S, Greiner F, Tadsen B and Piel A 2015 J. Phys. D: Appl. Phys. 48465203

[46] Greiner F et al 2018 Eur. Phys. J. D 7281

[47] Tadsen B, Greiner F and Piel A 2017 Phys. Plasmas $241-6$

[48] Platier B, Staps T J A, Schans M V d, IJzerman W L and Beckers J 2019 Appl. Phys. Lett. 115254103

[49] Haverlag M, Kroesen G M W, Bisschops T H J and de Hoog F J 1991 Plasma Chem. Plasma Process. 11 357-70
[50] Beckers J, Van De Wetering F M J H, Platier B, Van Ninhuijs M A W, Brussaard G J H, Banine V Y and Luiten O J 2019 J. Phys. D: Appl. Phys. 52034004

[51] van der Schans M, Platier B, Koelman P, van de Wetering F, Van Dijk J, Beckers J, Nijdam S and IJzerman W 2019 Plasma Sources Sci. Technol. 28035020

[52] Slater J C 1946 Rev. Mod. Phys. 18 441-512

[53] Biondi M A 1951 Rev. Sci. Instrum. 22 500-2

[54] Pozar D M 2005 Microwave Engineering (New York: Wiley)

[55] Van Ninhuijs M A W, Daamen K A, Beckers J and Luiten O J 2021 Rev. Sci. Instrum. 92013506

[56] Chow W and Rosenberg M 1994 IEEE Trans. Plasma Sci. 22 179-86

[57] Mitrani J M, Shneider M N, Stratton B C and Raitses Y 2016 Appl. Phys. Lett. 108054101

[58] Shneider M N 2015 Phys. Plasmas 22 1-5

[59] Stoffels W W, Stoffels E, Kroesen G M W and De Hoog F J 1995 J. Appl. Phys. 78 4867-72

[60] Bacal M 2000 Rev. Sci. Instrum. 71 3981-4006

[61] Küllig C, Dittmann K and Meichsner J 2010 Plasma Sources Sci. Technol. 19065011

[62] Park M, Na B, Kwak J G, Kim T S, Jung B, Huh S R and Jeong S H 2021 Phys. Plasmas 28023505

[63] Ossiander M et al 2018 Nature 561 374-7

[64] Lee D H, Pegg D J and Hanstrop D 1999 AIP Conf. Proc. 475 189-92

[65] Babilotte P and Vandevraye M 2017 J. Theor. Appl. Phys. 11 87-95

[66] Basha T S and Abbas A 1989 Eur. J. Phys. 10 151-6

[67] Cui C and Goree J 1994 IEEE Trans. Plasma Sci. 22 151-8

[68] Lieberman M A and Lichtenberg A J 2005 Principles of Plasma Discharges and Materials Processing (New York: Wiley)

[69] Tang X Z and Delzanno G L 2014 Phys. Plasmas 21123708 